\title{
THE ESTABLISHMENT OF AN ASTROMETRIC STANDARD REGION IN THE NGP: STAR CATALOGUE
}

\author{
L.K. PAKULYAK \\ Main Astronomical Observatory \\ Ukrainian Academy of Sciences \\ 252127 Kiev \\ USSR
}

ABSTRACT. The positions and proper motions catalogue in the area near the NGP is presented.

The astrometric standard region (AS) in the NGP is supposed to be a supplement to three standard regions in Praesepe, Pleiades and IC4756 clusters, completing the net of them on the northern sky available for observations at any station in any moment of time (Russell, 1984).

The adopted technique of AS derivation, as it exists now, allows the updating of the catalogue with new data.

AS is centered on the Coma cluster with centre coordinates of $12^{\mathrm{h}} 24^{\mathrm{m}}$ in RA and $+26^{\circ}$ in Dec. A square area of $6^{\circ} \times 6^{\circ}$ is covered with 16 plates of the double wide-angle astrograph $(\mathrm{F}=2 \mathrm{~m}, \mathrm{D}=$ $40 \mathrm{~cm}$ ). Centres of plates were displaced by $2^{\circ}$ in both coordinates. The average plate epoch is 1984.2. Plates were made to the west and to the east from the astrograph column for the elimination of the instrumental magnitude equation. Two exposures on every plate were made for this purpose also. The main principle of the star choice is a homogeneous density of stars both in general and in any magnitude interval. The catalogue includes objects up to $15^{\mathrm{m}}$. The average density of stars is 84 stars per sq. degree. The measuring accuracy is ranging 2.3 to $3.0 \mu \mathrm{m}$.

The plate reduction model was chosen with the Eichhorn-Williams statistical test and optimum models for each plate were fitted together with the determination of instrumental aberrations, namely the coma coefficient and the tangential distortion terms (Pakulyak, 1989).

Each plate was reduced separately with the optimum reduction model. Final positions were derived as the average of all images, weighted with generalised Shlesinger coefficients. This technique is the same as Shlesinger one but a non-linear reduction model is used. Generalised weights were calculated for every field star in relation to the reference stars configuration (Kiselev,1984).

First iteration mean star positions appeared to be sufficient because the second iteration gave only a little better accuracy (see Table 1) due to the "spreading" of corner plates errors on the whole star field.

Positional errors (internal convergence and reduction errors) for both coordinates which depend on the zone of overlap are given in the same table.

Proper motions were derived for 537 objects up to $13^{\mathrm{m}}$ and improved for 525 stars from the AGK3 list. The improvement performed with 4 catalogues employed: AGK2, AGK3, AS and the reduced 
Astrographic Catalogue (Oxford section) on the area $12^{\circ} \times 6^{\circ}$ near the NGP (Stock, Cova, 1983). The epoch of the last one is 1901.1 and overlap with AS region is near $50 \%$. Weighted equations for proper motion determinations together with positions were written for all catalogues and all AS plate images. The positional accuracy of these stars is of \pm 0.014 in RA and \pm 0 ". 19 in Dec. The accuracy of proper motions is $\pm 0.00035 \mathrm{~s} / \mathrm{yr}$ and $\pm 0.0047 / \mathrm{yr}$ in RA and Dec correspondingly. For field stars there were only AS and Oxford section positions; corresponding proper motion errors are of $\pm 0.00039 \mathrm{~s} / \mathrm{yr}$ and $\pm 0.0032 \mathrm{H} / \mathrm{yr}$.

The magnitude equation in proper motions in the whole star magnitude range is of 0.019 in RA and -0.011 in Dec for the AGK3 list stars and of 0.003 in RA and near zero in Dec for field stars and eliminated by three iterations.

Magnitudes for AGK3 stars were converted to the B-system by linear equations for stars,common with SAO catalogue. The mean accuracy is of $0^{\mathrm{m}} 13$. The same attempt for field stars failed because of errors worse than 0.64. That's why field stars magnitudes are given the same as in the Astrographic Catalogue.

The total number of objects catalogue contains is 13197 in magnitude range $6^{\mathrm{m}}$ to $15^{\mathrm{m}}$ with 1062 objects, which have proper motions.

Table 1. Positional accuracy of AS stars

\begin{tabular}{llllll}
$\begin{array}{l}\text { i-fold } \\
\text { overlap }\end{array}$ & $\begin{array}{l}\text { Number } \\
\text { of stars }\end{array}$ & $\begin{array}{l}\text { 1st iter.(Positional errors) } \\
\text { inter conv. }\end{array}$ & $\begin{array}{l}\text { 2 nd iteration } \\
\text { reduct. }\end{array}$ & inter.conv. & reduct. \\
\hline & & & & & \\
1 & 425 & 0.35 & 0.35 & 0.27 & 0.27 \\
2 & 329 & 0.21 & 0.18 & 0.19 & 0.14 \\
3 & 321 & 0.27 & 0.19 & 0.22 & 0.14 \\
4 & 1316 & 0.21 & 0.15 & 0.18 & 0.13 \\
6 & 467 & 0.19 & 0.13 & 0.15 & 0.11
\end{tabular}

\section{References}

Kiselev, A.A. (1984), Problemy astrometrii, pp.44-56.

Pakulyak, L.K. (1989), Kinematika i fizika neb.tel, 251, 2, pp.24-28.

Stock, J.S., Cova, S. (1983), Rev.Mexicana Astron.y Astrof., 251, 4, pp.233-260.

Russell, J.L. (1984), IAU Symposium 109, pp.697-703. 